\title{
Agrotóxicos: uma revisão de suas consequências para a saúde pública
}

\author{
Vivian Brusius Cassal', Letícia Fátima de Azevedo², Roger Prestes Ferreira ${ }^{3}$, \\ Danúbio Gonçalves da Silva ${ }^{4}$, Rogers Silva Simão ${ }^{5}$ \\ 1,2,3,4,5 Instituto de Desenvolvimento Educacional do Alto Uruguai - IDEAU -Campus Bagé, RS, Brasil
}

\section{Resumo}

Este estudo, por meio de uma revisão, objetivou relatar os perigos da utilização indiscriminada de agrotóxicos e suas consequências na sáude pública. Objetivo inspirado justamente pelo fato do Brasil estar entre os maiores consumidores de agrotóxicos do mundo e pelo impacto social e ambiental causado pelo uso desordenado destes. Assim, sabemos que os riscos não se limitam ao homem do campo, atingem os mananciais de água, o solo, o ar, os animais, podendo também os alimentos comercializados nas cidades apresentaram resíduos tóxicos. Parte dos agrotóxicos utilizados tem a capacidade de se dispersar no ambiente, e outra parte pode se acumular no organismo humano, trazendo diversos efeitos agudos e crônicos. Por fim, no mundo todo, os efeitos dos impactos ambientais vêm sendo percebidos. Em relação à saúde pública, o uso cada vez mais crescente desses compostos tem causado severos efeitos, sejam eles agudos ou crônicos, em vários trabalhadores, principalmente da área rural, embora outros setores também sejam afetados.

Palavras-chave: agrotóxicos, contaminação, saúde.

\begin{abstract}
This study, by means of a review aimed, to report the dangers of indiscriminate use of pesticides and its impact on public health. Objective inspired precisely by the fact that Brazil is among the largest consumers of pesticides in the world and the social and environmental impact caused by the use of these disordered. Thus, know that the risks are not limited to a farmer, reach the springs of water, soil, air, animals, and may also foods marketed in the cities showed toxic waste. Part of agrochemicals has the ability to disperse in the environment, and the other part can accumulate in the human body, bringing various acute and chronic effects. Finally, throughout the world, the effects of environmental impacts are being perceived. In relation to public health, the ever-increasing use of these compounds has caused severe effects, whether acute or chronic, in many workers, especially from rural areas, while other sectors are also affected.
\end{abstract}

Keywords: pesticides, pollution, health. 


\section{INTRODUÇÃO}

O uso de agrotóxicos tem se difundido na agricultura, principalmente, nos últimos 30 anos. Especificamente o Brasil, se tornou um dos maiores consumidores desses produtos xenobióticos, ficando atrás somente do Japão e dos Estados Unidos (DAMS, 2006). O Brasil encontrava-se entre os oito maiores consumidores de agrotóxicos do mundo (CARNEIRO \& ALMEIDA,s.d.). Há três anos o Brasil ocupa o primeiro lugar no ranking de consumo de agrotóxicos no mundo (ORTIZ, 2012). No Rio Grande do Sul o uso de agrotóxicos chega a quase o dobro da média nacional (CIGANA, 2013).

Em 2002, foram disponibilizados para o agricultor brasileiro 2.011 produtos formulados com registro no Ministério da Agricultura, dentre eles 655 herbicidas1, 556 inseticidas2, 259 acaricidas 3 e 58 nematicidas4 para o controle de pragas, doenças e ervas daninhas (SINDAG, 2005). Em 2010, de acordo com a Anvisa (2013) o mercado nacional movimentou cerca de U\$7,3 bilhões e representou 19\% do mercado global de agrotóxicos. Em 2008, o Brasil ultrapassou os Estados Unidos e assumiu o posto de maior mercado mundial de agrotóxicos.

Um terço dos alimentos consumidos cotidianamente pelos brasileiros está contaminado pelos agrotóxicos, segundo alerta feito pela Associação Brasileira de Saúde Coletiva (Abrasco), em dossiê lançado durante o primeiro congresso mundial de nutrição o World Nutrition Rio 2012 (ORTIZ, 2012).

Especificamente sobre a safra 2009/2010 foi destacado o uso de 85 milhões de litros de agrotóxicos no Rio Grande do Sul, o equivalente a 34 piscinas olímpicas cheias de veneno agrícola. É como se cada gaúcho, à época, utilizasse 8,3 litros de veneno a cada ano, no período analisado. $\mathrm{O}$ volume per capita gaúcho é bem superior ao nacional, que em 2011, a média do país foi de 4,5 litros por habitante (ABRASCO, 2013).

Outra constatação refere-se a existência de uma concentração do mercado de agrotóxicos em determinadas categorias de produtos. Os herbicidas, por exemplo, representaram $45 \%$ do total de agrotóxicos comercializados. Os fungicidas 5 respondem por $14 \%$ do mercado nacional, os inseticidas $12 \%$ e, as demais categorias de agrotóxicos, 29\% (ANVISA; UFPR, 2012).

O processo produtivo agrícola brasileiro está cada vez mais dependente dos agrotóxicos e fertilizantes químicos. A lei dos agrotóxicos (Lei Federal no 7.802, de 11 de julho de 1989, atualmente regulamentada pelo Decreto 4.074, de 4 de janeiro de 2002), define que essas substâncias são: "os produtos e os agentes de processos físicos, químicos ou biológicos, destinados ao uso nos setores de produção, no armazenamento e beneficiamento de produtos agrícolas, nas pastagens, na proteção de florestas, nativas ou implantadas, e de outros ecossistemas e também de ambientes urbanos, hídricos e industriais, cuja finalidade seja alterar a composição da flora ou da fauna, a fim de preservá-las da ação danosa de seres vivos considerados nocivos bem como substâncias e produtos empregados como desfolhantes, dessecantes, estimuladores e inibidores do crescimento". Essa definição exclui fertilizantes e químicos administrados a animais para estimular crescimento ou modificar comportamento reprodutivo (PORTAL EDUCAÇÃO, 2008).

Segundo dados da Agência Nacional de Vigilância Sanitária (Anvisa) e do Observatório da Indústria dos Agrotóxicos da Universidade Federal do Paraná, divulgados durante o $2^{\circ}$ Seminário sobre Mercado de Agrotóxicos e Regulação, realizado em Brasília (Distrito Federal), em abril de 2012, enquanto, nos últimos dez anos, o mercado mundial de agrotóxicos cresceu 93\%, o mercado brasileiro cresceu 190\%. Mato Grosso é o maior consumidor de agrotóxicos, representando 18,9\%, seguido de São Paulo (14,5\%), Paraná (14,3\%), Rio Grande do Sul (10,8\%), Goiás (8,8\%), Minas Gerais (9,0\%), Bahia (6,5\%), Mato Grosso do Sul (4,7\%) e Santa Catarina (2,1\%). Os demais estados consumiram 10,4\% do total do Brasil (IBGE, 2006; SINDAG, 2011; THEISEN, 2012).

Em relação às hortaliças, com base em dados disponíveis no Dossiê Abrasco (2013) o consumo de fungicidas atingiu uma área potencial de aproximadamente 800 mil hectares, contra 21 milhões de hectares somente na cultura da soja. Isso revela um quadro preocupante de concentração no uso de ingrediente ativo de fungicida por área plantada em hortaliças no Brasil, podendo chegar entre 8 a 16 vezes mais agrotóxico por hectare do que o utilizado na cultura da soja, por exemplo.

\footnotetext{
1 Pesticida utilizado na agricultura para o controle de ervas classificadas como daninhas.

2 Pesticida usado para exterminar insetos, destruindo ovos e larvas principalmente.

3 Pesticida usado no extermínio dos ácaros.

4 Pesticida usado para matar nematóides parasitas.

5 Pesticida que destrói ou inibe a ação dos fungos que geralmente atacam as plantas.
} 
Numa comparação simples, estima-se que a concentração de uso de ingrediente ativo de fungicida em soja no Brasil, no ano de 2008, foi de 0,5 litro por hectare, bem inferior à estimativa de quatro a oito litros por hectare em hortaliças, em média. Pode-se constatar que cerca de $20 \%$ da comercialização de ingrediente ativo de fungicida no Brasil é destinada ao uso em hortaliças. Dessa maneira pode-se inferir que o uso de agrotóxicos em hortaliças, especialmente de fungicidas, expõe de forma perigosa e frequente a saúde do consumidor, o ambiente e os trabalhadores à contaminação química por uso de agrotóxicos (ALMEIDA; CARNEIRO; VILELA, 2009).

Pesticidas, quando usados corretamente, causam pouco impacto adverso no meio ambiente. Entretanto, quando utililzado indiscriminadamente, sem as devidas precauções e cuidados em relação a manipulação, produção, estocagem e destino final, põe em risco não só o meio ambiente, mas também a saúde das pessoas que de alguma forma entram em contato com tais produtos. É evidente que traços de resíduos de pesticidas presentes no solo, água, ar e alimentos podem ser perigosos à saúde do homem e ao meio ambiente.

Com isso, o objetivo desta revisão é relatar os perigos da utilização indiscriminada de agrotóxicos na agricultura e suas consequências na sáude pública.

\section{REVISÃO DE LITERATURA}

\section{I IMPACTO NA SAÚDE PÚBLICA}

Elevados níveis de contaminação humana e ambiental têm sido encontrados em regiões agrícolas no Brasil. São vários os fatores que contribuem para essa estatística, como, por exemplo, a ampla utilização, o desrespeito às normas de segurança, a livre comercialização e a pressão exercida pelas empresas distribuidoras e produtoras. Faria et al. (2004) relatam que os principais fatores ocupacionais que evidenciam um risco aumentado para intoxicações são: aplicar agrotóxicos, reentrar na cultura após aplicação, usar equipamentos para trabalho com agrotóxicos mais que dez dias por mês e trabalhar com agrotóxicos em mais de uma propriedade.

Conforme a figura1 podemos evidenciar o ranking de alimentos de acordo com o percentual de amostras inadequadas para o consumo, segundo a Anvisa (2011).

Um terço dos alimentos consumidos cotidianamente pelos brasileiros está contaminado pelos agrotóxicos, segundo análise de amostras coletadas em todas as 26 Unidades Federadas do Brasil, realizadas pelo Programa de Análise de Resíduos de Agrotóxicos em Alimentos (PARA) da ANVISA (2011). Evidenciou-se que 63\% das amostras analisadas apresentaram contaminação por agrotóxicos, sendo que $28 \%$ apresentaram ingredientes ativos não autorizados (NA) para aquele cultivo e/ou ultrapassaram dos limites máximos de resíduos (LMR) considerados aceitáveis.

O caso mais grave é o do pimentão com $92 \%$ das amostras irregulares, contra $63 \%$ dos morangos, $57 \%$ do pepino, $54 \%$ das amostras de alface e $50 \%$ de cenoura. O tomate, que já esteve no topo do ranking, hoje, tem contaminação de $16 \%$. Foram observadas irregularidades em cerca de $30 \%$ das amostras analisadas de beterraba, mamão e abacaxi. O alimento que saiu ileso de agrotóxicos foi a batata que obteve resultado satisfatório em $100 \%$ das amostras analisadas.

Parte dos agrotóxicos utilizados tem a capacidade de se dispersar no ambiente, e outra parte pode se acumular no organismo humano, inclusive no leite materno. O leite contaminado ao ser consumido pelos recém-nascidos pode provocar agravos à saúde, pois os mesmos são mais vulneráveis à exposição a agentes químicos presentes no ambiente, por suas características fisiológicas e por se alimentar, quase exclusivamente com o leite materno até os seis meses de idade (CARNEIRO et al., 2012).

São inúmeros os estudos que associam o uso de agrotóxicos e seus efeitos nocivos na saúde humana (ALAVANJA, 1999; COLOSSO et al., 2003; PERES et al., 2003; SANTOS, 2003). Os efeitos agudos, conforme Mariconi (1986) aparecem durante ou após o contato da pessoa com o agrotóxico, podendo ser divididos em efeitos muscarínicos (brandicardia, miose,espasmos intestinais e brônquicos, estimulação das glândulas salivares e lacrimais); nicotínicos (fibrilações musculares e convulsões); e centrais (sonolência, letargia, fadiga, cefaléia, perda de concentração, confusão mental e problemas cardiovasculares).

Segundo Koifman e Hatagima (2003) um grande número de agrotóxicos apresenta atividade potencialmente capaz de desregular o equilíbrio endócrino de seres humanos e animais, sendo que a 


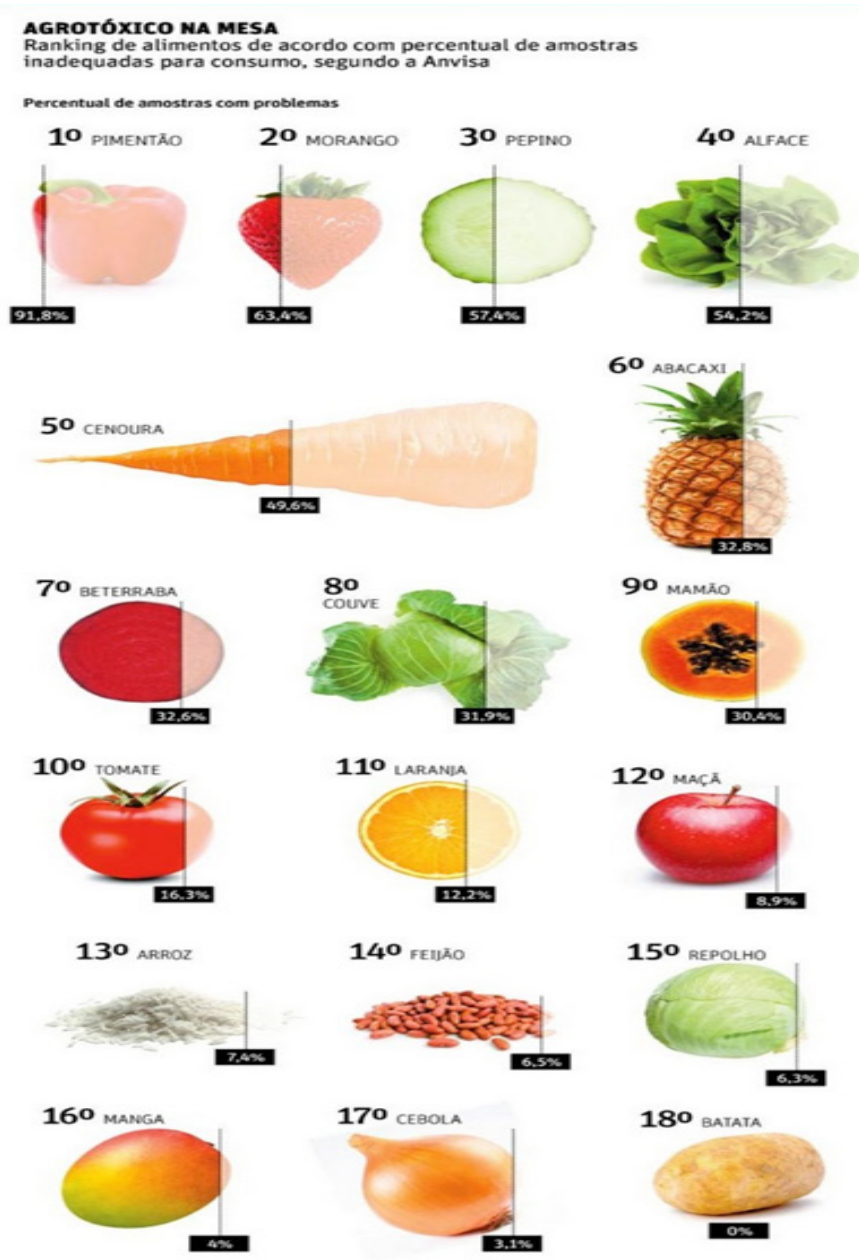

figura 1: ranking de alimentos de acordo com o percentual de amostras inadequadas para o consumo. Fonte: ANVISA (2011).

exposição a esses disruptores endócrinos estaria associada a cânceres, a modificação na razão entre sexos ao nascimento, infertilidade, más-formações congênitas no trato genital masculino e a modificações na qualidade do sêmen.

$\mathrm{O}$ uso indiscriminado de agrotóxicos tem resultado em intoxicações, em diferentes graus, de agricultores e de consumidores, tornando-se um problema de saúde pública. Apesar de vários estudos evidenciarem as graves consequências que estes podem implicar, ainda existem no Brasil alguns obstáculos que impedem o desenvolvimento de uma agricultura menos agressiva para as pessoas e para o meio ambiente (PIRES et al., 2005).

A exposição ocupacional aos agrotóxicos tem um forte impacto na saúde pública. Os efeitos mais bem documentados sobre a exposição ocupacional de trabalhadores rurais envolvem o sistema nervoso. Keifer e Mahurin (1997) observaram que as conseqüências neurotóxicas de uma exposição aguda de alto nível estão associadas a uma série de sintomas e defeitos na conduta neurológica e anormalidades na função nervosa. Os sintomas neurológicos menos severos incluem dor de cabeça, tontura, náusea, vômito e excessivo suor. Já os mais perigosos são o desenvolvimento de fraqueza muscular e bronquiespasmos, podendo progredir para convulsões e coma.

Bhatt et al. (1999) também notaram que a exposição a pesticidas pode estar associada ao aumento do risco de doenças neurodegenerativas, particularmente a Doença de Parkinson. Entre algumas das manifestações de intoxicação por agrotóxicos observadas em trabalhadores rurais estão a diminuição das defesas imunológicas, anemia, impotência sexual masculina, cefaléia, insônia, alterações da pressão arterial, alterações do humor e distúrbios do comportamento, como surtos psicóticos (LUNDBERG et al., 1997).

Segundo a Organização Mundial de Saúde (OMS), as doenças crônicas não transmissíveis 
(DCNT) foram responsáveis por $63 \%$ das mortes no mundo em 2008 e por $45,9 \%$ do volume global de doenças. A Organização prevê que o número de mortes deve crescer 15\% até 2020 em decorrência desses tipos de doença.

No Brasil, os casos de intoxicação por agrotóxicos apresentam grande subnotificação e descentralização de informações, decorrentes de fatores diversos: dificuldade de acesso dos agricultores às unidades de saúde, inexistência de centros de saúde em regiões produtoras importantes, dificuldade de diagnóstico e de relacionar os problemas de saúde com a exposição a agrotóxicos, escassez de laboratórios de monitoramento biológico e inexistência de biomarcadores precoces e/ou confiáveis. Para Menezes (2006) apesar das deficiências de registro, o Sistema Nacional de Informação Tóxico-Farmacológica (SINITOX) e o Sistema Nacional de Informação de Agravos Notificáveis (SINAN) são uma referência importante.

Embora o maior consumo de agrotóxicos ocorra nos países desenvolvidos, grande parte de envenenamentos e mortes causados por agrotóxicos ocorre nos países em desenvolvimento, sendo preocupantes os quadros de contaminação humana e ambiental observados no Brasil. Acredita-se que essa realidade possa estar associada à utilização desses produtos em excesso, à ocorrência de inadequados padrões ocupacionais e de segurança, ao desconhecimento dos riscos associados a sua utilização e conseqüente ineficiente uso de equipamentos de proteção individual, a elevados níveis de analfabetismo, à regulamentação e rotulagem insuficientes, a inadequadas ou inexistentes infra-estruturas para lavagem dos utensílios, ao manuseio inadequado dos resíduos e das embalagens, ao aproveitamento dos recipientes para armazenar alimentos e água, bem como à grande pressão comercial por parte das empresas distribuidoras e produtoras. Podem-se acrescentar também a existência de uma fiscalização precária do cumprimento das leis, uma deficiente assistência técnica ao homem do campo e a baixa atenção à saúde (CAMPANHOLA e BETTIOL, 2002; MOREIRAet al., 2002).

Como já mencionado anteriormente, o Brasil é o oitavo maior consumidor de agrotóxicos por hectare do mundo, sendo os herbicidas (utilizados na destruição de plantam que impedem o desenvolvimento da cultura desejada) e inseticidas (produto próprio para matar insetos) responsáveis por $60 \%$ dos produtos comercializados no país. No período compreendido entre 1992 e 2002 foram registradas no Estado do Mato Grosso do Sul 1.355 notificações de intoxicações provocadas pelo manuseio de agrotóxicos na agricultura, sendo destes, cerca de 500 casos decorrentes de ingestão voluntária (PIRES et al., 2005).

Levigard e Rozemberg (2004), em trabalho realizado em Nova Friburgo (RJ), a partir de entrevistas com profissionais da área da saúde, onde foram analisadas as formas de tratamento prestado às queixas de "nervos" dos agricultores. Os autores ressaltam o fato dos profissionais na área da saúde estarem preocupados com os hábitos da população no consumo indiscriminado de calmantes, caracterizando a automedicação. O uso de remédios sem prévia consulta com especialista, juntamente com o uso dos agrotóxicos utilizados nas lavouras, acaba por agravar o processo de intoxicação dos indivíduos.

Investigações no estado do Rio Grande do Sul também apontam os malefícios da utilização de agrotóxicos para o meio ambiente e população humana. Faria et al. (2004), em estudo realizado com trabalhadores rurais cultivadores da fruticultura dos municípios de Antônio Prado e Ipê, constataram que das famílias entrevistadas, 95\% informaram utilizar algum tipo de agrotóxico, $73 \%$ faziam uso regular de agrotóxicos na agricultura, e que, em média, $75 \%$ dos trabalhadores rurais relataram trabalhar regularmente com os agrotóxicos.

Os autores acima constaram que a prevalência de exposição agroquímica foi maior entre os homens, estes em $86 \%$ dos casos, enquanto em $68 \%$ de casos com mulheres. Entre os agricultores, $35 \%$ disseram nunca terem usado luvas, máscaras ou roupas de proteção. Em um grupo considerado pelos autores sem escolaridade esse índice foi maior. Também percebeu-se que, as intoxicações ocorreram, na maioria das vezes, entre outubro e janeiro, pois neste período devido às temperaturas elevadas, o organismo absorve mais as toxinas liberadas pelos agrotóxicos e também pelo fato das aplicações serem mais intensas nessa época do ano. A gravidade destas intoxicações foi considerada leve-moderada em $80 \%$ dos casos e como grave em $20 \%$ das ocorrências (FARIA et al., 2004).

Segundo o IBGE (2012), cerca de 70 milhões de brasileiros vivem em estado de insegurança alimentar e nutricional, sendo que $90 \%$ desta população consume frutas, verduras e legumes abaixo da quantidade recomendada para uma alimentação saudável. Segundo o Dossiê Abrasco (2012) superação deste problema, seria por meio da conversão do modelo agroquímico/tradicional e mercantil para um 
modelo de base agroecológica, baseado no controle social e na participação popular. No entanto, isso trata-se de decisão política, de longo prazo, onde a educação continuada e a pesquisa também deverão ser fortalecidas nessa perspectiva.

Apesar da grande importância das atividades agrícolas, há pouco interesse no estudo de aspectos da saúde e segurança na agricultura. Existe grande interesse em desenvolver novas tecnologias para aumento da produção na agropecuária, sem levar em consideração os impactos à saúde e à segurança do trabalhador (FRANK et al., 2004).

Diante do exposto, no consumo de alimentos vegetais, o primeiro cuidado é saber a procedência dos produtos, optando sempre pelos que possuem origem identificada. Segundo a Anvisa (2012), a identificação irá aumentar o comprometimento dos produtores em relação à qualidade dos alimentos. Optar por orgânicos e sazonais também são procedimentos recomendados pela a Anvisa para obter um produto livre de agrotóxicos. A lavagem correta dos alimentos e a retirada de cascas e folhas externas ajudam na redução dos resíduos de agrotóxicos presentes apenas nas superfícies.

\section{CONSIDERAÇÕES FINAIS}

No mundo todo, os efeitos dos impactos na saúde pública, bem como no meio ambiente vêm sendo percebidos. Em relação à saúde pública, o uso cada vez mais crescente desses compostos tem causado severos efeitos, sejam eles agudos ou crônicos, em vários trabalhadores, principalmente da área rural, embora outros setores também sejam afetados.

O produtor rural é/será diretamente afetado. Ele tem que ser, portanto, o primeiro a tomar providências, pois o seu ramo de atividade depende da adoção de práticas que conservem o meio ambiente e a sua própria saúde. É fato que para se produzir alimentos em larga escala, é indispensável o uso consciente dos agrotóxicos como uma ferramenta a mais para assegurar a proteção, contra baixas produtividades ou perdas de culturas.

Porém, o controle químico só deve ser empregado após aplicação de todos os métodos de controles disponíveis, para se evitar problemas toxicológicos tanto para o homem quanto para o meio ambiente. Relatórios recentes de institutos de pesquisa demonstram que, caso nada seja feito, o clima será alterado em proporções drásticas, prejudicando especialmente aqueles setores da economia que dependem da natureza, como é o caso da agricultura.

\section{REFERÊNCIAS}

ALMEIDA, V. S; CARNEIRO, F. F; VILELA, N. J. Agrotóxicos em Hortaliças: Segurança Alimentar e Nutricional riscos socioambientais e políticas públicas para a promoção da saúde. Tempus Actas de Saúde Coletiva, v.4, p.84-99, 2009.

ABRASCO - ASSOCIAÇÃO BRASILEIRA DE SAÚDE COLETIVA. Disponível em: < http: //www. abrasco.org.br/>. Acesso em: 22 dez. 2013.

ALAVANJA, M. C. Characteristics of persons who self reported a high pesticide exposure event in the Agricultural Health Study. Environ Res., 80:180-186, 1999.

ANVISA. Agência Nacional de Vigilância Sanitária. Programa de Análise de Resíduo de Agrotóxico em Alimentos (PARA), dados da coleta e análise de alimentos de 2010. Brasília: ANVISA, 2011. Disponível em: <http:// www.anvisa.gov.br>. Acesso em: 21 de dez. 2011.

Agrotóxicos. Disponível em: <http:// www.anvisa.gov.br/monografias/index. htm>. Acesso em: 08 jul. 2013.

2011. Disponível em: < http:// www.portal.anvisa.gov.br>. Acesso em: 10 jul. 2013. 
ANVISA; UFPR. Seminário de mercado de agrotóxico e regulação. Brasília: ANVISA. Acesso em: 11 abr. 2013.

BHATT, M. H.; ELIAS, D. M. A.; MANKODI, B. S. et al. Acute and reversible parkinsonism

due to organophosphate pesticide intoxication. Neurology, 52: 1.467.

BRASIL, 2010. Ministério da Saúde. Protocolo de Atenção à Saúde dos Trabalhadores Expostos a Agrotóxicos. Disponível em: <http:// www.portal.saude.gov.br/portal/arquivos/pdf/integra agrotoxicos.pdf $>$. Acesso em: 18 jul. 2013.

BRASIL, Decreto n. ${ }^{\circ} 4.074$ de 04 de janeiro de 2002. Regulamenta a Lei no $7.802 / 89$ (Lei Federal dos agrotóxicos). Brasília, Diário Oficial da União de 08/01/2002.

CAMPANHOLA, C.; BETTIOL, W. Panorama sobre o uso de agrotóxicos no Brasil. In: Ministério do Meio Ambiente (org.). Fórum Nacional de Secretários de Agricultura. Programa de Defesa Ambiental Rural - textos orientadores. 2002.

CARNEIRO,F.F.; ALMEIDA,V.E.S.e BRASIL é o país que mais usa agrotóxicos no mundo. Disponível em: $<$ http:// www.mg.gov.br $>$. Acesso em : 17 nov. 2013.

CARNEIRO, F. F.; PIGNATI, W.; RIGOTTO, R. M. et al. Dossiê ABRASCO - Um alerta sobre os impactos dos agrotóxicos na saúde. Parte 1 - Agrotóxicos, Segurança Alimentar e Nutricional e Saúde. Rio de Janeiro: ABRASCO, 2012.

CIGANA,C. Uso de agrotóxicos no Rio Grande do Sul chega quase ao dobro da média nacional. Disponível em: < http://www.zerohora.clickrbs.com.br $>$. Acesso em: 26 nov. 2013.

COLOSSO, C.; TIRAMANI, M.; MARONI, M. Neurobehavioral effects of pesticides: state of theart. Neurotoxicology, 24, p.577-591, 2003.

DAMS, R. I. Pesticidas: usos e perigos à saúde e ao meio ambiente. 2006. Disponível em: <http:/www. periodicos.univille.br/index.php/RSA/article/download/89/139P. Acesso em: 08 jun. 2013.

DOSSIÊ ABRASCO (Associação Brasileira de Saúde Coletiva). Um alerta sobre os impactos dos Agrotóxicos na Saúde. Parte 1 - Agrotóxicos, Segurança Alimentar e Nutricional e Saúde. Disponível em: <http://www4.planalto.gov.br $>$. Acesso em: 10 jul. 2013.

FARIA, N. M. X.; FACHINI, L. A.; FASSA, A. G.; TOMASI, E. Trabalho rural e intoxicações por agrotóxicos. Caderno de Saúde Pública, Rio de Janeiro, v.20, n.5, p.1298-1308, 2004.

FAO - FAOSTAT. Data base Results. [on line]. Disponível em: < http://apps.fao.org>. Acesso em: 08 jul. 2013.

FILIZOLAH. F.; FERRACINI V. L., SANS L. M. A., et al. Monitoramento e avaliação de risco de contaminação por pesticidas em água superficial e subterrânea na região de Guairá. Pesquisa Agropecuária Brasileria. 37(5), p.659-667, 2002.

FRANK, A. L.; MCKNIGHT, R.; KIRKHORN, S. R.; GUNDERSON, P. Issues of agricultural safety and health.Annual Review of Public Health. Palo Alto, v. 25, p. 25-45, 2004.

IBAMA. Produtos agrotóxicos e afins comercializados em 2009 no Brasil. 2009. Disponível em: < http:// www.ibama.gov.br/qualidade - ambiental/wp content/files / Produtos _ Agrotoxicos 
_Comercializados_Brasil_2009.pdf>.Acesso em: 17 jun. 2013.

IBGE - INSTITUTO BRASILEIRO DE ESTATÍSTICA E GEOGRAFIA. Censo agropecuário do Brasil. 2006. Disponível em: <http://www.ibge.gov.br>.Acesso em: 10 jun. 2013.

. Disponível em: <http//www.ibge.gov.br>.Acesso em: 10 set.. 2013.

JEPPSON, L. R.; KEIFER, H. H.; BAKER, E. W. Mites injurious to economic plants. Berkeley: UniversityofCalifornia Press, 614 p., 1975.

KEIFER M., MAHURIN R. Chronic neurological effects of pesticides overexposure. Occup. Med. 12: 291304, 1997.

KOIFMAN, S.; HATAGIMA A. Disruptores endócrinos no ambiente: efeitos biológicos potenciais (Editorial). Revista Brasileira de Mastologia, 13 (1) p.9-11, 2003.

LEVIGARD, I. E.; ROZEMBERG, B. A interpretação dos profissionais de saúde acerca das queixas de "nervos" no meio rural: uma aproximação ao problema das intoxicações por agrotóxicos. Caderno de Saúde Pública, Rio de janeiro, v.20, n.6, p.1515-1524, 2004.

LUCHINI, L. C.; ANDRÉA, M. M. de. Dinâmica de agrotóxicos no ambiente. In: BRASIL. Ministério do Meio Ambiente (org.). Fórum Nacional de Secretários de Agricultura. Programa de Defesa Ambiental Rural - textos orientadores. 2002.

LUNDBERG, I., HOGBERG, M., MICHEISEN, H. et al. Effects of long term organophosphate exposure on neurologicalsymptoms, vibration sense and tremor among South African farmer workers. Occup. Environ. Med. 54: 343-350, 1997.

MARICONI, F.A.M. Inseticidas e seu Emprego no Combate às Pragas. Ed. Agron. Ceres. São Paulo, 607p., 1986.

MARTINS JUNIOR, O. P. A dinâmica dos agrotóxicos no meio ambiente. 2002. Disponível em: <http:// www.mp.go.gov.br/.../a dinamica dos agrotoxicos no meio ambiente>. Acesso em: 13 jul. 2013.

MARTINS, M. D., FERNANDES, C. S., VALENTE, J. T. Water contamination by pesticides. Case study: pesticides research in the Lower Cávado River Basin. In: WORLDWATER CONGRESS, 4, 2004, Marrakesh. [Anais eletrônicos...] Marrakesh: IWA, 2004. 1CD-ROM.

MENEZES, C. T. Método para priorização de ações de vigilância da presença de agrotóxicos em águas superficiais: um estudo em Minas Gerais. Dissertação de Mestrado. Universidade federal de Minas Gerais, 2006.117p.

MOREIRA, J. C.; JACOB, S. C.; PERES, F.; et al. Avaliaçãointegrada do impacto do uso de agrotóxicos sobre a saúde humana em comunidade agrícola deNova Friburgo, RJ. Ciência \& Saúde Coletiva, v. 7, n. 2, 2002. p. 299-311.

OPAS/OMS - ORGANIZAÇÃO PAN-AMERICANA DA SAÚDE/ORGANIZAÇÃO MUNDIAL DA SAÚDE. Manual de vigilância da saúde de populações expostas a agrotóxicos. Brasília:1996. Disponível em: < http://www.opas.org.br/sistema/arquivos/livro2.pdf>. Acesso em: 06 jun. 2013.

ORTIZ, F. Um terço dos alimentos consumidos pelos brasileiros está contaminado por agrotóxicos. Disponível em: < http://www.noticias.uol.com.br/saude/ultimas-noticias/redacao/2012/05/01/um-terco-dos -alimentos- consumidos- pelos- brasileiros- esta-contaminado-por-agrotoxicos.htm >. Acesso em: $20 \mathrm{dez}$. 2013. 
PERES, F.; MOREIRA J. É veneno ou é remédio? Agrotóxicos, saúde e ambiente. Rio de Janeiro: Editora Fiocruz. 2003. 384 p.

PIRES, D. X.; CALDAS, E. D.; RECENA, M. C. P. Uso de agrotóxicos e suicídios no estado do Mato Grosso do Sul, Brasil. Caderno de Saúde Pública, Rio de Janeiro, v.21, n.2, p.598-605, 2005.

PORTAL EDUCAÇÃO. 2008. Disponível em: $<$ http:// www.portaleducacao.com.br /enfermagem/artigos/810/definicao- e classificacao\#ixzz2oA9IKF3W>. Acesso em: 21 dez. 2013.

SANTOS, S. L. Avaliação de parâmetros da imunidade celular em trabalhadores rurais expostos ocupacionalmente a agrotóxicos em Minas Gerais. Dissertação de Mestrado. Departamento de Bioquímica e Imunologia, Instituto de Ciências Biológicas, Universidade Federal de Minas Gerais, Belo Horizonte. 2003.

SINDAG - SINDICATO NACIONAL DAS INDÚSTRIAS DE DEFENSIVOS AGRÍCOLAS. Uso de defensivos é intensificado no Brasil. 2005. Disponível em: $<$ http://www.sindag.com.br/noticia.php?News $\underline{\text { ID }=2278>}$. Acesso em: 29 jun. 2013.

Vendas de defensivos agrícolas são recordes e vão a US\$ 8,5 bi em 2011. 2011. Disponível em: $<$ http://www.sindag.com.br/noticia.php?News ID=2256>. Acesso em: 01 jul. 2013.

TADEO J. L., SÁNCHEZ-BRUNETE C., BEATRIZ A. B., et al. Analysis of pesticide residues in juice and beverages. Critical Rev. Analytical Chem. 34, p.121-131, 2004.

THEISEN, G. O mercado de agroquímicos. Disponível em: <http://www.cpact.embrapa.br/eventos/2010/ met/palestras/28/281010_Painel3_Giovani_THEISEN.pdf>. Acesso em: 17 jun. 2013. 